\title{
Un modelo de creación de mercado con trading de alta frecuencia
}

\author{
Daniel Hernández Hernández \\ Katherine Sánchez Casas ${ }^{* *}$
}

* Docente Centro de Investigación en Matemáticas cimat, Guanajuato (México).dher@ cimat.mx ** Departamento de Estadística Universidad Nacional de Colombia, Bogotá (Colombia). ksanchezc@unal.edu.co

Fecha de recepción: 15 de mayo de 2016.

Fecha de aceptación: 5 de junio de 2016.

Para citar este artículo:

Hernández Hernández, D. y Sánchez Casas, K. (2016). Un modelo de creación de mercado con trading de alta frecuencia. ODEON, 11, pp. 123-142. DOI: https://doi.org/10.18601/17941113.n11.06 



\section{Introducción}

Para ser rico no es necesario ser hijo de ricos o ganarse la lotería, el mundo financiero está implementando aproximadamente desde 1999 una tecnología bursátil que permite ganar millones de dólares en milisegundos. Dicha tecnología está sustituyendo agentes humanos por agentes robotizados que operan miles de veces más rápido que los humanos para efectuar millones de órdenes compraventa por segundo, permitiendo ganar más dinero.

Esta tecnología se denomina trading de alta frecuencia, o High Frequency Trading (HFT), el cual emplea sofisticados programas computacionales que prevén cómo van a funcionar los mercados desde una técnica cuantitativa; el algoritmo analiza datos del mercado en busca de oportunidades de colocación, observando los parámetros del mercado u otra información en tiempo real, con esa información se traza un mapa en el que se determina: el momento adecuado de negociación,el precio y la cantidad. Orientando la división de órdenes a través del tiempo y a los lugares de mercado, y la elección de la estrategia de inversión en órdenes límite y de mercado, estos algoritmos son implementados en un tiempo bastante corto.

La posibilidad de entrar a los mercados directamente y de realizar órdenes de posiciones en milisegundos ha causado un rápido crecimiento de este tipo de operaciones en el total del volumen del mercado. Se calcula que el trading de alta frecuencia supone más del $60 \%$ de las operaciones de capital en EE.UU., del $40 \%$ en Europa y del 10\% en Asia. Inicialmente, el HFT fue desarrollado en el contexto de los mercados de renta variable y en los últimos años se ha extendido a opciones, futuros, ETFS (cambio de fondos negociados), divisas y materias primas.

En el artículo se considera un agente que participa en el mercado de valores por medio del trading de alta frecuencia; dicho agente es pequeño y no causa impactos en el precio de mercado. En esta dinámica, el agente está expuesto a tres riesgos principales, que son el riesgo de inventario, el riesgo de selección adversa y el riesgo de ejecución.

En la construcción de la estrategia de creación de mercado intervienen tres factores: el precio, el spread y el inventario (Labadie y Fodra, 2013). El precio medio evoluciona de manera estocástica, el Bid-Ask Spread evoluciona de manera aleatoria y cambia de estado con la introducción de órdenes de compra y venta de los participantes en el mercado.

En la primera sección de este artículo se presentan algunas definiciones y 
características de HFT, se expone el modelo de creación de mercado, finalmente se presentan las conclusiones y futuros trabajos de investigación.

\section{Qué es HFT y cuáles son sus características}

Para hablar del trading de alta frecuencia o HFT es necesario presentar su definición.

La Comisión Europea HFT como:

Sistema de negociación que analiza a gran velocidad datos o señales del mercado y lanza o actualiza, como reacción de dicho análisis, un gran número de órdenes en un periodo de tiempo muy corto, prácticamente en milisegundos. Por ello, HFT no consistiría tanto en una estrategia de negociación, sino en la utilización de tecnologías avanzadas para llevar a cabo estrategias de negociación tradicionales, como sería el arbitraje y la propia creación de mercado. ${ }^{1}$

HFT está caracterizado por ejecutar órdenes intradiarias en periodos muy cortos de tiempo, medido en fracciones de segundos, y procura mantener los inventarios cercanos a cero al finalizar el día (Kearns, Kulesza y Nevmyvaka, 2010). HFT hace uso de recursos computacionales sofisticados y de alta velocidad, normalmente bajo la figura de hospedaje ${ }^{2}$, que ofrecen las bolsas de valores; estas envían una gran cantidad de órdenes, que son ejecutadas en fracciones de segundo o, incluso, son canceladas, buscando cerrar todas las posiciones al final de la jornada (Kirilenko, Kyle, Samadi y Tuzun, 2011).

HFT es un término genérico que hace referencia a un conjunto diverso de estrategias cuyo denominador común es que son algorítmicas y tienen una baja latencia (la baja latencia se refiere a la rápida ejecución de órdenes; es decir, muy poco tiempo transcurrido entre el momento en el que la orden es enviada y en ser ejecutada) para obtener una ventaja en el mercado (Prewitt, 2012).

${ }^{1}$ Considerando 44 de la Propuesta de Directiva del Parlamento Europeo y del Consejo relativa a los mercados de instrumentos financieros.

${ }^{2}$ El hospedaje se define como la ubicación de servidores contiguos a los de la bolsa, de tal suerte que se pueda minimizar la latencia en el flujo de información. 


\subsection{Características de HFT}

Los agentes que hacen uso del trading de alta frecuencia (HFT) son firmas propietarias de las mesas de negociación en bancos de inversión, fondos de cobertura, etc., que sobre la base de estas estrategias tienen la capacidad de generar grandes cantidades de operaciones en periodos corto tiempo (Cvitanic y Kirilenko, 2010)

Las empresas propietarias dedicadas a la negociación de alta frecuencia están caracterizadas por:

- Uso de equipos computacionales equipados de gran capacidad (software y hardware) equipados con programas informáticos sofisticados de alta velocidad, con los que se tiene acceso a la información de mercado, generadores de enrutamiento, ejecución y cancelación de órdenes.

- El uso de los servicios de coubicación con los cuales se permite situar sus servidores físicamente próximos al sistema central de procesamiento, ganando así unos microsegundos en el tiempo de latencia ya que el estar cerca de la fuente de intercambios afecta la velocidad de la reacción en fracciones de segundo, lo que proporciona una valiosa ventaja cuando se negocia en el mercado, en la medida en que están dispuestos a pagar millones de dólares por este servicio (Cartea y Penalva, 2011).

- Presentación de las numerosas órdenes que son canceladas poco tiempo después de la presentación; el objetivo del ingreso de dichas órdenes es capturar el spread compra-venta antes que otros jugadores, spread que generalmente es muy pequeño y comúnmente está en décimas, pero que por la alta velocidad y cantidad de operaciones es un negocio rentable.

- Plazos muy cortos para establecer y liquidar posiciones; HFT son estrategias de negociación algorítmica basadas en la explotación del procesamiento y la mayor velocidad de ejecución para obtener ganancias comerciales durante un tiempo muy corto, normalmente medido en segundos, en su mayoría de pocos minutos, y desde luego menos de un día.

- Generalmente finalizan el día de negociación sin posiciones abiertas trading intra-day, por lo que no implica la necesidad de un capital importante o una gestión de riesgo más profunda que si se tomaran posiciones con un horizonte de tiempo más amplio. 


\subsection{Estrategias de HFT}

Existen diversos tipos de estrategias de HFT, cada una con diferentes peculiaridades según la firma propietaria, las cuales se describirán de manera concisa; entre las principales estrategias se encuentran: creación de mercado, arbitraje estadístico, detección de liquidez, manipulación de precios y otras estrategias; en esta categoría se agruparon varias que no pertenecen a las anteriores.

- La estrategia de creación de mercado emite constantemente órdenes límite de compra y de venta competitivas, de esta manera proporciona liquidez al mercado y su ganancia promedio está dada por el margen oferta/demanda, (spread bid/ask) (Hendershott, Jones y Menkveld, 2011); esta, junto con la inyección de liquidez, proporciona una ventaja en la captación del spread, porque las operaciones rápidas son menos propensas a ser afectadas por los movimientos de precios.

- Las estrategias de arbitraje estadístico consisten en la búsqueda de activos relacionados, o en el mismo activo que negocia en diferentes mercados (p.e. futuro y subyacente, acción común y preferencial, entre los derivados y sus activos subyacentes, entre los fondos Exchange-traded (ETF) y sus valores constitutivos, o simplemente entre los activos estadísticamente correlacionados en el mismo mercado) que estructuralmente tienden a moverse al unísono; cuando encuentran diferencias entre dichos activos, los arbitrajistas compran el más barato y venden el más caro ganando la diferencia. Debido a su velocidad, HFT puede participar en el arbitraje en tiempos extremadamente cortos, y con diferencias de precios enormemente pequeñas, generando rendimientos.

- En las estrategias denominadas detección de liquidez, los algoritmos HFT intentan identificar ganancias de las acciones de otros operadores de gran tamaño; por ejemplo, mediante la agregación de múltiples puntos de datos desde diferentes intercambios y en busca de patrones característicos en variables como la profundidad de la orden. Dichos algoritmos pueden establecer una orden límite oculta, o un gran comerciante que intenta entrar o salir de una posición. El objetivo de estas tácticas es beneficiarse de las variaciones del precio creado por otros comerciantes, de manera que pueden comprar justo antes de la ejecución de grandes órdenes de otros comerciantes; una variante de esta estrategia consiste en tratar de detec- 
tar y predecir los patrones de comportamiento de los demás operadores y explotar su impacto en el mercado.

- Estrategias de manipulación del mercado. Algunos de los métodos utilizados por los operadores de alta frecuencia no son tan limpios ya que causan problemas al mercado y, en cierto sentido, son ilegales; estas estrategias disfrazan sus ofertas evitando que otros participantes en el mercado descubran sus intenciones comerciales. Una de las características más significativas de los operadores de HFTS es la manera como usan las cancelaciones para estimular el mercado y extraer información valiosa. Tres de estas tácticas son conocidas como: stuffing, smoking y spoofing.

- Stuffing: consiste en generar deliberadamente un gran número de cotizaciones que los competidores tienen que procesar, pero que estas firmas ignoran ya que ellos las han generado, de esa manera obtienen una ganancia en el tiempo de proceso, permitiéndoles obtener beneficios de las cotizaciones de sus competidores que estarán retrasadas debido al procesamiento de las órdenes generadas por ellos; en consecuencia, la maquinaria del mercado también sufrirá retrasos e incluso puede colapsar debido al excesivo volumen de mensajes.

- Smoking: este procedimiento consiste en la publicación de órdenes límite seductoras para atraer a los comerciantes menos rápidos, entonces ellos revisan rápidamente estas órdenes en términos menos generosos, con la esperanza de ejecutar con rentabilidad contra el flujo entrante de órdenes de mercado de los comerciantes menos rápidos.

- Spoofing o suplantación, consiste en el envío de una gran cantidad de órdenes límite en un corto periodo de tiempo; estas no están destinadas a ser ejecutadas, su objetivo es la manipulación de precios. Esta actividad ilegal distorsiona la forma del libro de órdenes, con el objetivo explícito de engañar a otros comerciantes.

- En la categoría otras estrategias se encuentran:

- La estrategia estructural escudriña la existencia de cotizaciones desactualizadas, estas son órdenes límites de compra o venta que se encuentran a un precio superior o inferior, respectivamente, que aún son vigentes y que no han sido retiradas. 
- La estrategia direccional consta de posiciones descubiertas y apuestas agresivas. Se basan en una anticipación estimada de la dirección del movimiento de precios intradiarios.

- El arbitraje de latencia involucra el comercio en tiempos por debajo de un segundo entre el momento en el que los precios del mercado se mueven y cuando los creadores de mercado actualizan sus precios de cotización.

\section{Modelo de creación del mercado}

\subsection{Creación de mercado}

El modelo de creación de mercado considera un agente en un mercado de valores, que presenta títulos de valores de compra y venta a un determinado precio de manera continua; este agente se denomina creador de mercado (MM por sus siglas en inglés, market maker).

En la página oficial de la National Futures Asociation - Estados Unidos (NFA), este agente es definido como un profesional encargado de negociar, siendo la contrapartida de sus clientes.

La denominación de creador de mercado obedece a que provee liquidez continua y regular a sus clientes. De manera que si un cliente desea comprar, esta orden es absorbida por el creador de mercado, es compensada con la orden de otro cliente, quien actúa como intermediario ante las posibles faltas de liquidez del mercado (caso en el que un inversor quiera comprar o vender y no haya una contraparte en el mercado que quiera vender o comprar), de manera que crea un mercado entre sus clientes.

Los creadores de mercado participan y juegan un papel importante en mismo, en particular para mejorar la liquidez de valores o stocks y de esta manera promover el crecimiento a largo plazo en el mercado. Como proveedor de liquidez, estos agentes reciben una compensación: compran a un precio inferior (bid) y venden a un precio superior (ask). La diferencia entre el precio de cotización al que los participantes del mercado están dispuestos a comprar (ask o precio de demanda) o vender un activo (bid o precio de oferta), se conoce como el Bid-Ask Spread.

Debido a que cada agente puede comprar o vender una acción en un momento dado, el spread representa los beneficios de estos. La amplitud o el tamaño del 
spread varía esencialmente en función de la liquidez y transparencia del mercado. Si hay un mayor número de vendedores y compradores compitiendo, hay más liquidez y, en consecuencia, se reduce la amplitud del spread. Si todos los participantes tienen acceso a la misma información sobre el precio (mayor transparencia) se reduce el spread puesto que para atraer más clientes entran en competencia los ofertantes y los agentes pueden encontrar una mejor oferta.

Las características propias que poseen las estrategias del creador de mercado es que no son direccionales, es decir no hay una ganancia asegurada debido a que el precio del activo puede subir o bajar. Generalmente, no guardan posiciones durante la noche, y no sostienen activos riesgosos al final del día de negociación.

Otro atributo importante es que mantienen su inventario o posición del activo de riesgo en un nivel mínimo de existencias, o cercano a cero durante el día de negociación, y frecuentemente equilibran sus posiciones en diferentes mercados, gracias al uso de algoritmos de alta frecuencia de negociación para el envío de órdenes.

Un creador de mercado compite con otros creadores para comprar o vender acciones, cuando el precio de una acción aumenta, los creadores subirán sus precios de compra, causando un movimiento ascendente. De esta manera, un creador de mercado está expuesto a tres principales riesgos, que son riesgo de inventario, riesgo de selección adversa y riesgo de ejecución.

El riesgo de inventario es el riesgo de sostener una posición corta o larga de un activo riesgoso, es decir es la posibilidad de pérdida asociada a las variaciones de los precios de los activos. El riesgo de selección adversa hace referencia a que el precio del mercado tenga un desvío desfavorable desde el punto de vista del creador de mercado, cuando este haya asumido una posición. El riesgo de ejecución surge por la incertidumbre de ejecución de órdenes límite, en caso de no ser ejecutadas o ser parcialmente ejecutadas.

Para familiarizar al lector con un vocabulario de frecuente uso durante el artículo, se presentan definiciones de los términos órdenes de mercado y órdenes límite, las cuales son las herramientas de negociación de activos de los creadores de mercado.

\subsection{Estrategias de creación de mercado}

La mayoría de intercambios de capital modernos son conducidos por órdenes de mercado, entendiendo "orden" como una instrucción de compra o venta ingresada en un mercado por un comerciante, que puede ser sobre un portafolio 
propio o de representación de un cliente, sobre un activo, especificando cantidad y precio según el caso.

En este mercado el precio resulta de la operación de un libro de órdenes o limit order book (LOB). En el LOB se almacenan a un lado todas las órdenes de compra (también conocidas como ofertas) y en el otro lado todas las de venta (conocidas como demandas).

Según el mercado y la autorización del regulador, existen distintos tipos de órdenes; en el desarrollo de este artículo se consideran dos tipos: órdenes límite y órdenes de mercado.

- Órdenes límite: una orden límite permite fijar un precio para vender o comprar una determinada cantidad de un activo, en algún momento futuro. Este tipo de órdenes son de ejecución y cancelación gratuita.

En el caso de compra, la orden indica el precio máximo que se está dispuesto a pagar, por lo que el precio límite introducido generalmente es inferior al actual precio de cotización. El precio más alto de una orden límite de compra se denomina mejor precio de la oferta (bid price).

En el caso de venta, la orden indica el precio mínimo que se está dispuesto a recibir, por tanto el precio límite introducido generalmente es superior al precio actual de cotización. El precio más bajo especificado en el libro de órdenes para una orden de venta se denomina precio de demanda (ask price).

En el momento en el cual un agente somete su orden límite para el intercambio, esta es añadida a la orden de libros límite; si hay contraparte disponible a dicho precio o a un precio mayor se ejecuta la porción de la cantidad disponible y el resto quedará pendiente en el libro de órdenes. No obstante, debe tenerse en cuenta que la ejecución de órdenes límite se basa en la regla de prioridad (precio/hora) del libro de órdenes límite, luego en una orden límite se da prioridad primero al precio, y a continuación en función del tiempo.

- Órdenes de mercado: son aquellas órdenes que se introducen en el mercado sin precio de compra o venta previamente fijado. El objetivo de este tipo de órdenes es que se ejecuten lo antes posible, de manera que se liquidan a los precios existentes en ese momento en el mercado, sin ninguna limitación. Esta clase de órdenes pueden ser ejecutadas a distintos precios, puesto que tienen prioridad en la ejecución inmediata y en la totalidad de 
la ejecución de ser posible, no en el precio; estas órdenes generan costos de presentación.

El creador de mercado tiene como opciones negociar por medio del envío de órdenes límite (figura 1) fijando posiciones; con estas órdenes el precio es establecido con antelación; sin embargo, la ejecución de este tipo de órdenes es incierta, el agente también puede negociar mediante órdenes de mercado, que son de ejecución inmediata, pero costosas ya que se emiten al precio de mercado y generan costos de presentación.

Con el anterior panorama, el creador de mercado puede presentar órdenes límite con mejores cotizaciones (oferta agresiva) (figura 1), aumentando el precio de compra con respecto a la mejor oferta actual (bid price), y disminuyendo su precio de venta (demanda agresiva) (figura1), con referencia a la mejor demanda actual (ask price). Así el agente se enfrenta a la disyuntiva entre la espera de ejecución de órdenes límite a los mejores precios actuales, o presentar órdenes límite con precios agresivos que se ejecutarán más rápidamente, pero a un precio menos favorable.

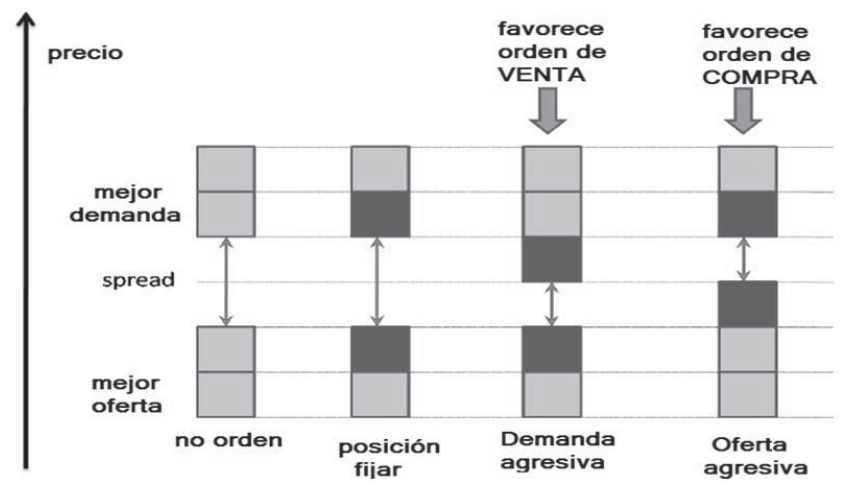

Figure 1: Estrategias de creación de mercado con órdenes límite.

El Bid-Ask Spread es usado por un creador de mercado para controlar su inventario y como compensación del riesgo de selección adversa.

Con la presentación de órdenes límite agresivas el Creador de Mercado captura órdenes de mercado de agentes que aún no están dispuestos a negociar a los precios de cotizaciones de oferta (bid price) y demanda (ask price). De este modo, el creador de mercado pierde dinero con los comerciantes informados, 
pero cubre esa pérdida haciendo ruido (es decir, los comerciantes no informados pagan el spread en cada transacción).

Con el objetivo de construir una estrategia de creación de mercado, deben tenerse en cuenta tres factores: el precio, el spread y el inventario. El precio suele ser el precio medio, es decir, el promedio entre los precios de mercado actuales de oferta y demanda. En la siguiente sección se formaliza el modelo de creación de mercado estudiado.

\subsection{Configuración del modelo}

Para introducir la característica principal del libro de órdenes LOB, se fija un espacio de probabilidad filtrado $(\Omega, \Im, \mathbb{F}, \mathbf{P})$, con una filtración $\mathbb{F}=\left(\mathcal{F}_{t}\right)_{t \geq 0}$ que satisface las condiciones usuales; de esta manera, todas las variables aleatorias y procesos estocásticos están definidos en $(\Omega, \Im, \mathbb{F}, \mathbf{P})$.

El precio medio $P_{t}$ del activo evoluciona de manera estocástica; en el artículo se asume que el precio medio evoluciona de acuerdo con un movimiento browniano geométrico dado por:

$$
d P_{t}=\mu P_{t} d t+\sigma P_{t} d W_{t} .
$$

Donde: $W_{t}$ es un movimiento browniano o proceso de Wiener y $\mu$ representa la variación porcentual, o porcentaje de deriva, y $\sigma$ es el porcentaje de volatilidad; estas dos cantidades son constantes. Si se tiene un valor inicial $P_{t}=P_{0}$, la solución de la ecuación es dada por la siguiente expresión analítica:

$$
P_{t}=P_{0} \exp \left[\left(\mu-\frac{\sigma^{2}}{2}\right) t+\sigma W_{t}\right] \text {. }
$$

El Bid-Ask Spread del libros de órdenes es resultado del comportamiento de los agentes en el mercado y evoluciona de manera aleatoria, en el que la observación futura solo depende del presente y no del pasado, por lo tanto es ajustada por una cadena de Markov discreta con valores finitos; sus valores son múltiplos de un número finito $\delta>0$, y salta en un tiempo aleatorio.

Para su modelación se define una cadena de Markov estacionaria y discreta sobre el espacio de probabilidad filtrado $(\Omega, \Im, \mathbb{F}, \mathbf{P})$, junto con un conjunto $\mathbb{S}$, no pp. $123-142 \cdot$ n. $^{\circ} 11 / 2016$ 
vacío y finito. Luego, la sucesión de variables aleatorias $\left(\hat{S}_{n}\right): \Omega \rightarrow \mathbb{S}, n \in \mathbb{N}$ define el proceso de Markov realizado por el Bid-Ask Spread, cuyo espacio de estados es un conjunto finito $\mathbb{S}=\delta \mathbb{I}_{m}$, con $\mathbb{I}_{m}:=\{1,2, \ldots, m\}, m \in \mathbb{N}-\{0\}$.

Ya que el conjunto de estados $\mathbb{S}$ es finito, la matriz de transición asociada a la cadena está dada por $\left(\rho_{i j}\right)_{1 \leq i, j \leq M}$, de manera que la probabilidad de transición es:

$$
\begin{aligned}
\rho_{i j} & =\mathbf{P}\left[\hat{S}_{n+1}=j \delta \mid \hat{S}_{n}=i \delta\right] \\
\rho_{i i} & =0
\end{aligned}
$$

independiente de $N$ y representa el spread en tiempo aleatorio.

Puesto que la cadena de Markov del Bid-Ask Spread cambia de estado con la introducción de órdenes de compra y venta de los participantes en el mercado, la cadena salta en tiempo aleatorio; de este modo, el parámetro que conduce dicho proceso variará con el tiempo de manera no homogénea, por tanto, se modela con un Proceso Poisson no homogéneo, con tasa $\lambda(t)$ y se denota con $\left(N_{t}\right)$.

El proceso spread en tiempo calendario $\left(\mathcal{S}_{t}\right)$, evoluciona de forma estocástica y determina el cambio de $\hat{S}$ en el tiempo $N$, es decir:

$$
S_{t}=\hat{S}_{N_{t}}, t \geq 0 .
$$

Dicho proceso $\left(\mathcal{S}_{t}\right)$ es ajustado con una cadena de Markov de tiempo continuo no homogénea, cuya matriz de intensidad está dada por:

$$
R(t)=\left(r_{i j}(t)\right)_{1 \leq i, j \leq m}= \begin{cases}r_{i j}(t)=\lambda(t) \rho_{i j} & \text { para } i \neq j \\ r_{i i}(t)=-\sum_{i \neq j} r_{i j}(t) & \text { para } i=j .\end{cases}
$$

la cual modela el parámetro de intensidad del tiempo de salto de la cadena; se asume que $S$ y $P$ son independientes.

El ingreso de órdenes de mercado de compra y venta materializa órdenes límite de venta y compra, respectivamente; por tanto, se requiere un proceso que enumere las órdenes límite ejecutadas tanto de venta como de compra. El número 
de órdenes límite de venta se expresa con $N^{a}$ y el número de órdenes límite de compra $N^{b}$; como se aprecia, dichos eventos son el resultado de variaciones estocásticas, de manera que los parámetros del proceso que representan la intensidad por unidad de tiempo con la cual ocurren los eventos, son procesos estocásticos, modelados por procesos de Cox, también conocido como un proceso de Poisson doblemente estocástico o proceso Poisson mixto.

Luego, $N^{a}$ y $N^{b}$ son procesos de Cox que representan procesos de conteo específicos, con procesos de intensidad aleatorios.

\section{Estrategias con órdenes límite y de mercado}

Como se mencionó en la sección de estrategias de creación de mercado, el agente puede negociar con órdenes límite y de mercado para manejar los riesgos a los que se encuentra expuestos.

Como se ilustra en la figura 2, un creador de mercado puede presentar órdenes límite a los siguientes precios:

$\mathrm{Bb}$ : conocido como Bid price, representa el mejor precio actual de oferta, esto es: $P_{t}^{b}=P_{t}-\frac{S_{t}}{2}$.

$\mathrm{Bb}+$ : simboliza el precio de oferta agresivo, esto es: $P_{t}^{b+}=P_{t}^{b}+\delta=P_{t}-\frac{S_{t}}{2}+\delta$, es decir aquí aumenta su precio de compra en el tiempo $t$.

Ba: denominado Ask price, indica el mejor precio actual de demanda, esto es: $P_{t}^{a}=P_{t}+\frac{S_{t}}{2}$.

Ba-: representa el precio de demanda agresivo, esto es: $P_{t}^{a-}=P_{t}^{a}-\delta=$ $P_{t}+\frac{S_{t}}{2}-\delta$, es decir baja el precio de venta en el tiempo $t$.

Donde: $S_{t}$ denota el spread al tiempo $t$.

Formalizando lo anterior, las estrategias de orden límite son modeladas por un proceso control predecible de tiempo continuo, denotado como:

$$
\alpha_{t}^{\text {make }}=\left(Q_{t}^{b}, Q_{t}^{a}, L_{t}^{b}, L_{t}^{a}\right) ; \quad t \geq 0 .
$$

Donde: 


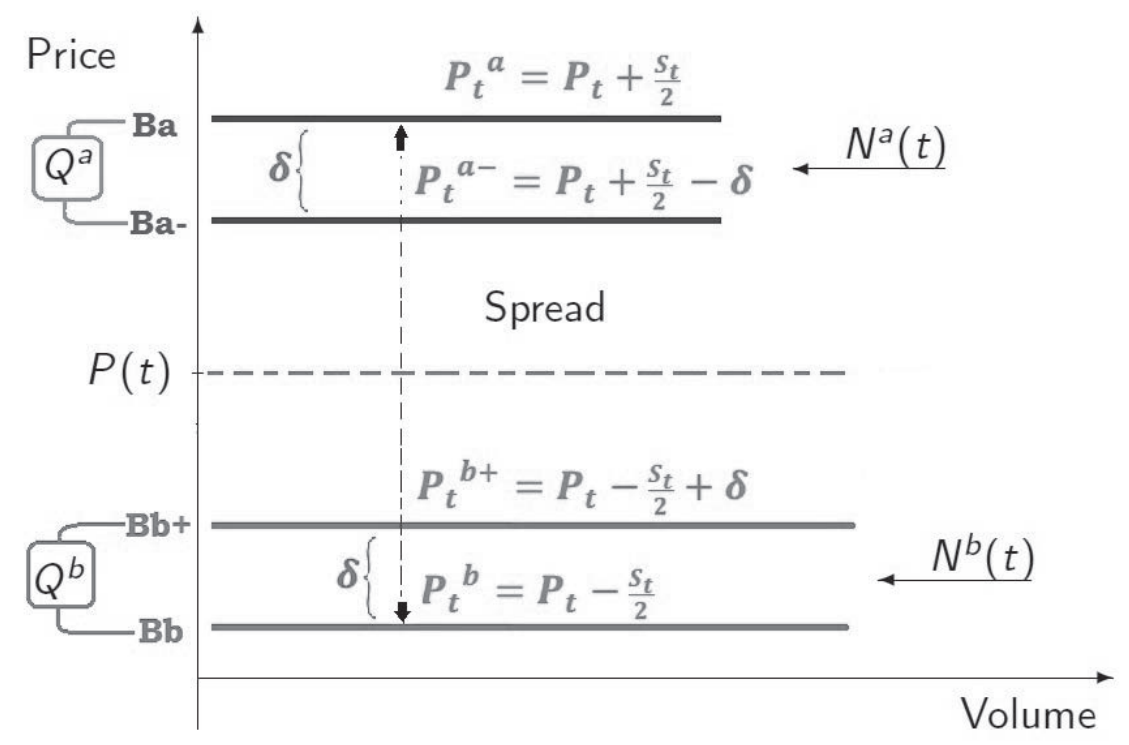

Figure 2: estrategias con órdenes límite.

- $\left(L^{b}, L^{a}\right)$ : representa el tamaño de órdenes límite de compra y venta, evaluadas en $[0, \ell]^{2}, \ell>0$

- $\left(Q^{b}, Q^{a}\right)$ : simboliza posibles cotizaciones de precios de compra o venta en cada tiempo $t$, esta es evaluada en $Q=Q^{b} \times Q^{a}$ con $Q^{b}=\{B b, B b+\}$ у $Q^{a}=\{B a, B a-\}$.

Se debe observar que estas cotizaciones son dependientes del tiempo $t$, sin embargo, para simplificar su expresión se omite $t$.

Los posibles precios en cualquier tiempo $t$ son denotados, respectivamente, como: $\pi^{b}\left(Q_{t}^{b}, P_{t}, S_{t}\right)$ y $\pi^{b}\left(Q_{t}^{b}, P_{t}, S_{t}\right)$ y están dados por:

$$
\begin{gathered}
\pi^{b}\left(q^{b}, p, s\right)= \begin{cases}p-\frac{s}{2} & \text { si } q^{b}=B b \\
p-\frac{s}{2}+\delta & \text { si } q^{b}=B b+\end{cases} \\
\pi^{a}\left(q^{a}, p, s\right)= \begin{cases}p+\frac{s}{2} & \text { si } q^{a}=B a \\
p+\frac{s}{2}-\delta & \text { si } q^{a}=B a-\end{cases}
\end{gathered}
$$


Las estrategias de orden límite $\alpha^{\text {make }}=\left(Q^{b}, Q^{a}, L^{b}, L^{a}\right)$, integrado con el dinero en efectivo, indicado como $X$, y el inventario o número de acciones sostenidas por el creador de mercado $Y$ mantienen la siguiente dinámica:

$$
\begin{gathered}
d Y_{t}=L_{t}^{b} d N_{t}^{b}-L_{t}^{a} d N_{t}^{a}, \\
d X_{t}=\pi^{a}\left(Q_{t}^{a}, P_{t}, S_{t}\right) L_{t}^{a} d N_{t}^{a}-\pi^{b}\left(Q_{t}^{b}, P_{t}, S_{t}\right) L_{t}^{b} d N_{t}^{b},
\end{gathered}
$$

Esta ecuación indica que el capital en el tiempo corresponde al precio obtenido por las compras, disminuido con el precio pagado en las ventas durante el periodo de negociación.

Puesto que el agente tiene permitido colocar órdenes de mercado que son liquidadas inmediatamente, estas estrategias evolucionan en tiempo discreto, por lo tanto son modeladas con un control de tipo impulso, dado por:

$$
\alpha^{\text {take }}=\left(\tau_{n}, \zeta_{n}\right)_{n \geq 0},
$$

en el cual $\left(\tau_{n}\right)$ es una secuencia creciente de tiempos de paro y representa los tiempos en el que el agente o creador de mercado decide invertir en órdenes de mercado, y las variables aleatorias $\zeta_{n}, n \geq 1$ son $\mathcal{F}_{\tau_{n}}$ medibles, se evalúan en $[-e, e]$, con $e$ tamaño de la orden de mercado.

Si $\zeta_{n} \geq 0$, son el número de acciones adquiridas al mejor precio de venta si $\zeta_{n}<0$, serán el número de acciones vendidas al mejor precio de compra.

Luego el cambio del inventario en tiempo discreto $\tau_{n}$, en el que el agente decide invertir en órdenes de mercado, es dado por:

$$
Y_{\tau_{n}}=Y_{\tau_{n}^{-}}+\zeta_{n}
$$

La expresión (6) indica que el cambio en el inventario en el momento de inversión en órdenes de mercado estará dado por el inventario obtenido en la anterior inversión en órdenes de mercado, aumentado con el número de acciones adquiridas al mejor precio de venta o disminuido por el número de acciones vendidas al mejor precio de compra. 
De forma semejante a la evolución del inventario con las inversiones en órdenes de mercado en el tiempo $\tau_{n}$, también se obtiene un cambio en el capital establecido por la siguiente relación:

$$
X_{\tau_{n}}=X_{\tau_{n}^{-}}-c\left(\zeta_{n}, P_{\tau_{n}}, S_{\tau_{n}}\right),
$$

donde $c\left(\zeta_{n}, P_{\tau_{n}}, S_{\tau_{n}}\right)=\zeta_{n} p_{\tau_{n}}+\left|\zeta_{n}\right| \frac{S_{\tau_{n}}}{2}$ simboliza la función de costo pagada, cuando pasa una orden de mercado de tamaño $\zeta_{n}$, con un spread observado $s_{\tau_{n}}$ y un precio medio observado $p_{\tau_{n}}$, con una cantidad fija $\varepsilon$. Resultando:

$$
X_{\tau_{n}}=X_{\tau_{n}^{-}}-\left(\zeta_{n} p_{\tau_{n}}+\left|\zeta_{n}\right| \frac{s_{\tau_{n}}}{2}+\varepsilon\right)
$$

Esta expresión (7) indica que el cambio en el capital al realizar inversiones en órdenes de mercado está dado por el capital alcanzado en la inversión anterior aumentado con el costo recibido de las acciones vendidas o disminuido con el valor pagado de las acciones compradas, cuando se tiene el spread observado $s_{\tau_{n}}$ dados en el momento $\tau_{n}$, y el precio medio observado $p_{\tau_{n}}$.

El conjunto de todas las estrategias de órdenes límite y órdenes de mercado se denota por $A$, dado por $\alpha=\left(\alpha^{\text {make }}, \alpha^{\text {take }}\right)$.

\section{Conclusiones}

Los inversores que hacen uso del trading de alta frecuencia HFT tienen una posición relativamente favorable en términos de ventajas de velocidad sobre el resto de participantes del mercado, una ventaja informativa (rápido acceso y análisis de la información de mercado) y una ventaja de velocidad de presentación en la negociación (la transmisión de baja latencia de los pedidos y ágiles modificaciones en las decisiones de negociación anteriores).

En el artículo se aplicacan conceptos del área de probabilidad para la modelación de un problema de aplicación real en el marco financiero, como lo es la creación de mercado en el contexto de HFT. Desde el campo de la probabilidad el precio medio fue modelado con un movimiento browniano geométrico, el proceso Bid-Ask Spread se modeló por medio de una cadena de Markov discreta con valores finitos; el tiempo de salto de la cadena fue modelado por un proceso Poisson no homogéneo y la ejecución de órdenes límite de venta y compra se modeló con procesos de Cox.

Dentro de los aportes del modelo adoptado se halla el control de las fuentes de riesgo a las que está expuesto el creador de mercado en la dinámica de su 
negociación, las cuales son el riesgo de selección adversa, el riesgo de inventario y el riesgo de ejecución.

Otra gran ventaja de este modelo es dar la alternativa al creador de mercado de publicar órdenes límite pasivas a las mejores cotizaciones de oferta/demanda (compra/venta), o presentar órdenes límite agresivas con respecto a las mejores ofertas y demandas, dando prioridad a la ejecución, que es un tema crucial en transacciones de alta frecuencia. Por otro lado, este modelo puede ser utilizado para modelar la evolución aleatoria del precio medio, los cambios de estado del Bid-Ask Spread por la introducción de órdenes de compra y venta de los participantes en el mercado y el tiempo de salto de este proceso, también incorpora las ejecuciones de órdenes límite de compra y venta.

Como trabajos de investigaciones futuras sería interesante la aplicación de este modelo a datos reales o simulados del mercado de valores, en el cual el creador de mercado pueda maximizar la utilidad de los ingresos esperados en un horizonte corto de tiempo, controlando su inventario, sujeto a que al final de la negociación su inventario debe ser cero, tal como sucede en el trading de alta frecuencia. 


\section{Referencias}

Avellaneda, M. y Stoikov, S. (s.f.). High Frequency Trading in a Limit Order Book. Quantitative Finance, 8 (3), 217-224.

Basawa, I. y Prakasa, R. (1980). Statistical Inference for Stochastic Process. Academic Press Inc.

Berkowitz, S. A., Logue, D. E. y Noser, E. A. (1988). The total cost of transactions on the NYSE. The Journal of Finance, 43 (1), 97-112.

Bertsekas, D. (2005). Dynamic Programming and Optimal Control. United States of America: Athena Scienific.

Bickel, P. (2001). Mathematical Statistics. United States of America: Prentice Hall.

Cartea, A., Jaimungal, S. y Ricci, J. (2011). Buy low sell high: a high frequency trading perspective. Preprint SSRN.

Cartea, A. y Penalva, J. (2011). Where is the Value in High Frequency Trading? Banco de España.

Cvitanic, J. y Kirilenko, A. (2011). High Frequency Traders and Asset Prices.

Durrett, R. (1999). Essentials of Stochastic Processes. United States of America: Springer.

Guilbaud, F. y Pham, H. (2013). Optimal high frequency trading with limit and market orders. The Journal of Finance, 13 (1), 79-94.

Hendershott, T., Jones, C. M. y Menkveld,A. J. (2011). Does algorithmic trading improve liquidity? The Journal of Finance, 66 (1), 1-33.

Karlin, S. y Pinsky, M. A. (2012). An Introduction to Stochastic Modeling. United States of America: Elsevier.

Kearns, M., Kulesza, A. y Nevmyvaka, Y. (2010). Empirical limitations on high frequency trading profitability. arXiv preprint arXiv:1007.2593.

Kirilenko, A., Kyle, A. S., Samadi, M. y Tuzun, T. (2011). The flash crash: The impact of high frequency trading on an electronic market. Manuscript, U. of Maryland. 
Labadie, M. y Fodra, P. (2013). High-frequency market-making with inventory constraints and directional bets. Quantitative Finance.

Lehalle, C.-A. (2013). Market Microstructure Knowledge Needed for Controlling an Intra-Day Trading Process. arXiv preprint arXiv:1302.4592.

Lehmann, E. y Casella, G. (1998). Theory of Point Estimation. New York: Springer.

Matt, P. (2012). High-Frequency Trading: Should Regulators Do More?

Moller, J. y Plenge, W. R. (2004). Statisitical Inference and Simulation for Spatial Poit Processes. United States of America: Chapman, and Hall//Crc.

Ortega, J. (2013). Notas procesos estocásticos. México: CIMAT.

Pliska, S. R. (1997). Introduction to mathematical finance. Oxford: Blackwell Publishers.

Ross, S. M. (2006). Simulation. United States of America: Elsevier.

Shao, J. (2003). Mathematical Statistics. New York: Springer.

Xu, J. (2013). Optimal Strategies of High Frequency Traders. 